\title{
A rare association: ankylosing spondylitis and a genetic disease
}

\author{
Andreea Alexandra Nicola ${ }^{1}$, Madalina Greere ${ }^{2}$, Adelina Lucretia Birceanu ${ }^{3}$, \\ Dinu Valentin Balanescu' ${ }^{1}$ Madalina Duna ${ }^{1}$, Denisa Predeteanu' ${ }^{1}$ \\ ${ }^{1}$ Department of Internal Medicine and Rheumatology, \\ "Sf. Maria" Clinical Hospital, Bucharest, Romania \\ 2Department of Gastroenterology, "Sf. Maria" Clinical Hospital, Bucharest, Romania \\ ${ }^{3}$ Department of Anatomopathology, "Sf. Maria" Clinical Hospital, Bucharest, Romania
}

ABSTRACT
Ankylosing spondylitis (AS) is a chronic systemic inflammatory disease that affects the axial skeleton and some-
times the peripheral joints, leading to the development of bone bridges and ankyloses with impaired joint mobility
and quality of life. The HLA B27 antigen, which occurs in approximately $97 \%$ of patients, is an important risk factor
and also a diagnostic element to consider. The typical onset of the disease is in the 3rd-4th decade of life; juve-
nile onset of AS under 16 years is associated with the predominant involvement of peripheral joints and multiple
complications (coxitis, acute anterior uveitis) which influence the evolution of the disease under treatment being
related with a negative prognosis.
Noonan syndrome is a genetic disease with dominant autosomal transmission characterized by a small stature
and other phenotypic features associated with congenital heart defects, especially pulmonary stenosis and atrial
septal defect. Multiple genes within the RAS subfamily involved in various cellular signaling pathways such as
signal transmission via mitogen-activated protein kinases are responsible for the occurrence of the disorder. Dif-
ferent hematological diseases such as myeloproliferative syndrome and neoplastic disease, particularly affecting
the lung, may be correlated with Noonan syndrome.
We present the case of a young patient with juvenile onset AS and Crohn's disease who has Noonan syndrome
with operated pulmonary stenosis and septal atrial defect, the association of these diseases bringing together
cumulative complications that required multiple therapies and surgical interventions with strict monitoring.
Keywords: juvenile-onset ankylosing spondylitis, Noonan syndrome, Crohn's disease,
multidisciplinary health care

\section{INTRODUCTION}

Affecting predominantly the sacroiliac and spinal joints, ankylosing spondylitis (AS) is the prototype of axial spondyloarthritis group which includes, depending of radiographic involvement of the joints, 2 forms of disease: non-radiographic AS in which the joint lesion is diagnosed using MRI of sacroiliac joint and spine and AS in which the joint lesion is diagnosed using X-ray of the sacroiliac joint. HLA B27 antigen is positive in over $97 \%$ of the patients and it is a disease risk marker and a positive diagnostic element $(1,2)$. Depending on the form of onset of the AS, there are two categories: juvenile - onset ankylosing spondylitis (JOAS) with manifestations before the age of 16 years and adult - onset ankylosing spondylitis (AOAS) with manifestations after the age of 16 years, these differentiations being useful in many aspects such as symptomatology, progression and evolution of the disease. In the JOAS, peripheral joints (especially ankles, knees, hips and shoulders) are more commonly affected clinically and radiologically, and these patients are more prone to hip arthroplasty, while in the AOAS the disease primarily affects the axial skeleton (3). Because of the different articulation structure, in the case of JOAS, the diagnosis is delayed, and clinical progression is faster and more severe, with impairment in functionality. At the same time, the prevalence of uveitis is higher in juvenile form. In the AOAS, the patients have a typical clinical picture with involvement of sacroiliac joints and lumbar spine; the appearence of acute anterior uveitis is less frequent and it can be independent of the activity of the spinal disease (4). 
The striking relationship between inflammatory bowel disease (IBD) and AS has been recognized for many years: up to $10 \%$ of IBD patients develop AS, and, vice versa, IBD commonly develops in patients primarily diagnosed with AS. As both have an important underlying genetic heritability, it has been suggested that the two diseases could have an overlapping set of predisposing genes such as HLA B27 (5).

Noonan syndrome was first described in 1883, but was named after Jaqueline Noonan who noticed that children with a rare type of heart defect called pulmonary valve stenosis often had a characteristic physical appearance with short stature, webbed neck, wide-spaced eyes, and low-set ears. She presented her first paper on the subject in 1963, and after several more papers and recognition, the condition was officially named Noonan syndrome in 1971 (6). It is a genetic disease with dominant autosomal transmission that is characterized by specific facies, low stature, developmental delay, cardiac abnormalities and skeletal malformations. Facial features include widely spaced eyes, light colored eyes, low set ears, a short neck, and a small lower jaw. Heart problems may include pulmonary valve stenosis. The sternum may be either protruded or clogged while the spine may be abnormally curved. Intelligence is often normal (7). Noonan syndrome may be caused by a mutation in any of several genes, and can be classified into subtypes based on the responsible gene. It is typically inherited in an autosomal dominant manner, but many cases are due to a new mutation and are not inherited from either parent (8). It is one of several RASopathies, the underlying mechanism for which involves a problem with a cell signaling pathway. The cause of $20-30 \%$ of Noonan syndrome cases remains unknown (9).

\section{CASE REPORT}

We present the case of a 33-year-old male, known with JOAS, admitted for clinical and paraclinical re-assessment to continue biological treatment with golimumab (Simponi). Upon presentation, the patient experienced intense pain in the left shoulder, at the insertion of the muscles on the antero-superior iliac spine, at the lumbar spine and bilateral knee pain requiring non-steroidal antiinflammatory drugs (NSAIDs).

The rheumatologic illness began in 1999 with pain in the small joints of the hands, later on lumbar spine, knees and other joints pain were added. Under the guidance of the local rheumatologist, the HLA B27 antigen was tested and the patient was diagnosed with AS. The recommended treatment at that time included sulfasalazine and NSAIDs without any favorable response. In addition, after 3 months, the patient presented sanguinolent diarrhea, which is why he interrupted the treatment based on his own initiative. Subsequently, after another treatment with sulfasalazine, methotrexate and meloxicam, he gave up this therapeutic regimen in May 2006 due to the appearance of sanguinolent diarrhea, vomiting, headache, arrhythmias, dyspnea and chills. Since June 2006, the patient has been enrolled in the Sf. Maria Clinical Hospital, Bucharest, Romania, where he was treated with Solu Medrol (methylprednisolone sodium succinate) pulse therapy. At this point, for the first time, a biological treatment was considered. Since January 2007, adalimumab (Humira) 40 mg, 1 ampoule every 2 weeks and methylprednisolone in the mild dose were given, with an initial favorable progression for 4 years (2007-2011). After 4 years, the patient presented secondary non-responsiveness to adalimumab. From 2011, the patient started treatment with etanercept (Enbrel) $50 \mathrm{mg}, 1$ ampoule per week and NSAIDs (diclofenac, meloxicam) under which the inflammatory syndrome and painful joint symptoms persisted. In 2013 the patient underwent a left hip prosthesis for coxitis with osteonecrosis. At the same time under etanercept (Enbrel) treatment, the patient suffered two episodes of uveitis in the same eye, which is why this treatment was switched with infliximab biosimilar (Remsima). In 2017, 2 infusions of Remsima were given, the second with an allergic reaction. Later, treatment with certolizumab (Cimzia) $200 \mathrm{mg}, 1$ ampoule every 2 weeks was indicated for a 3-month period, but his condition didn't improve at this time either, leading to, in March 2018, a second intervention, a right hip prosthesis, also for coxitis with osteonecrosis. In 2017, due to multiple episodes of sanguinolent diarrhea and persistent anemia (hemoglobin, 8 $\mathrm{g} / \mathrm{dl}$ ) associated with intense inflammatory syndrome (ESR $111 \mathrm{~mm} / \mathrm{h}$, CRP level over $100 \mathrm{mg} / \mathrm{dL}$ ), a colonoscopy was performed. This procedure highlighted suggestive elements for Crohn's Disease (inflammatory infiltration in the lamina propria, segmented focal encryption) and budesonide (Budenofalk) treatment was recommended, currently with a good response. It should be mentioned that during the evolution of the disease the patient presented significant leukocytosis, up to 30,000 leukocytes/mmc, which is why in November 2018 hema- 
tological evaluation was assessed, the bone marrow having a histopathological appearance of non-specific myeloid hyperplasia.

Considering small stature, typical phenotypic traits (characteristic facies with hypertelorism, low ears implantation and curly hair, pectus ecvinus, webbed neck) in a patient operated at 6 years old for atrial septal defect and pulmonary stenosis, the diagnosis of Noonan syndrome was established at 14 years old (Fig. 1).

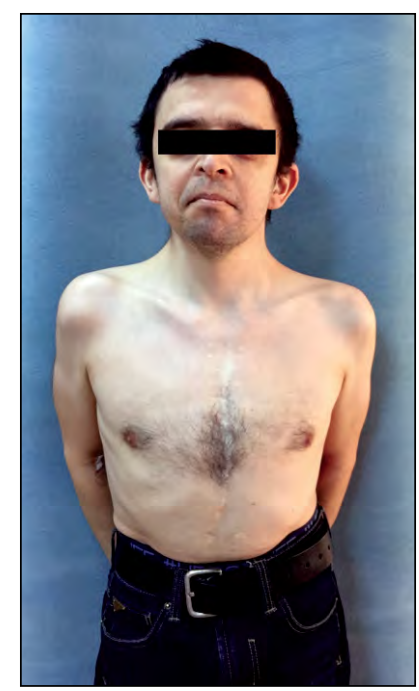

FIGURE 1. Typical characteristics of Noonan syndrome
The clinical examination at the current presentation reveals the typical characteristics for Noonan Syndrome: small stature $(150 \mathrm{~cm})$, thoracic postoperative scars. The AS evaluation shows the occiput-wall distance $=1 \mathrm{~cm}$, tragus-shoulder distance $=3 \mathrm{~cm}$, inspir-expir diameter $=3 \mathrm{~cm}$, finger-soil distance $=40 \mathrm{~cm}$, positive Schoeber maneuver; peripheral joints are without modifications, with normal functionality (Fig. 2).

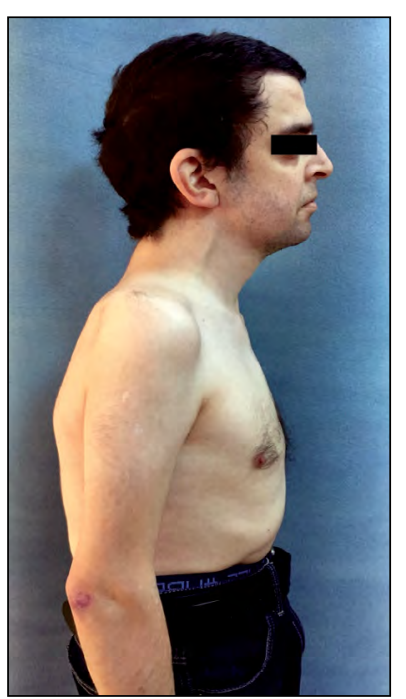

FIGURE 2. Typical characteristics of axial AS

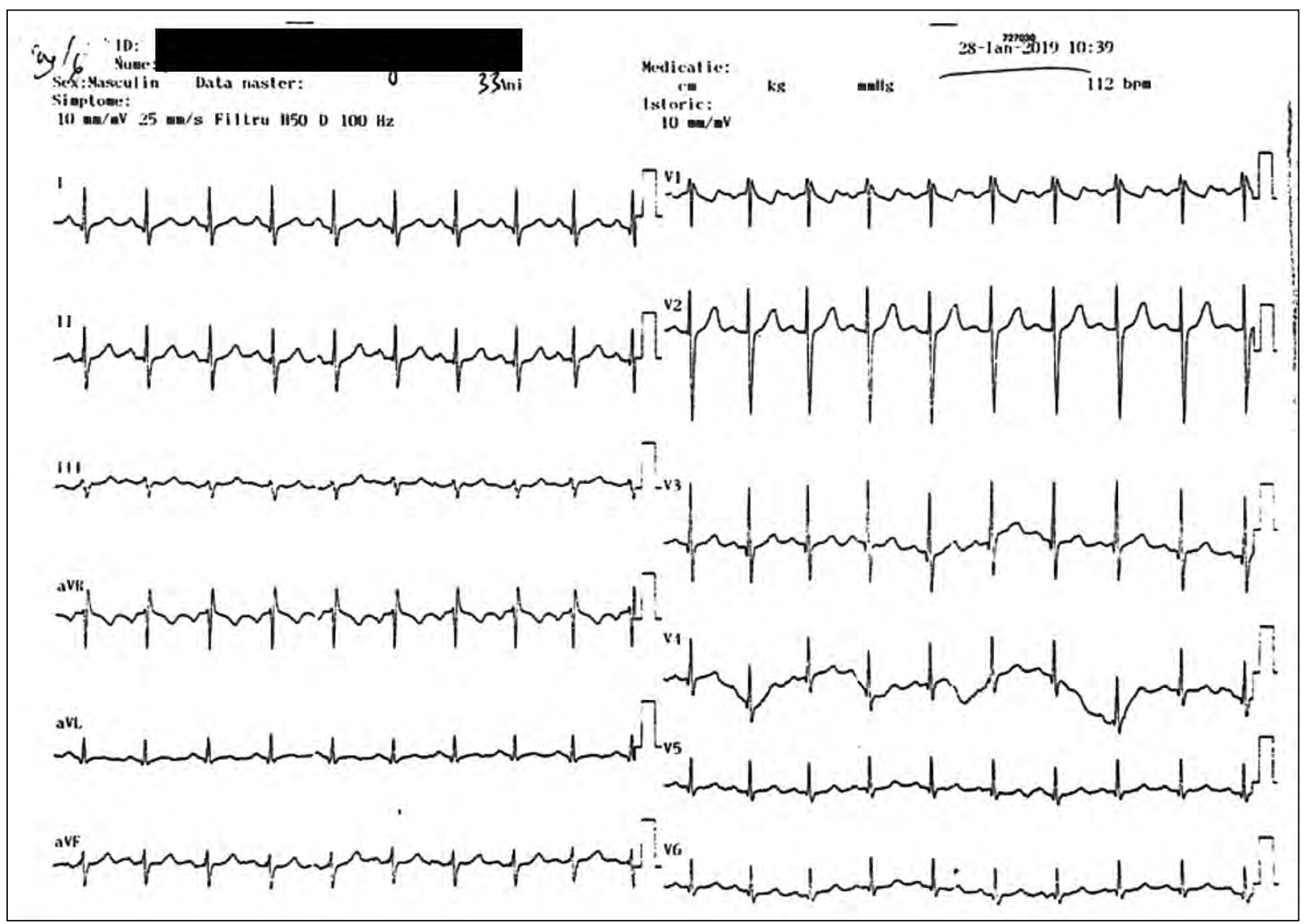


ECG shows verticalized heart, sinus rhythm and a minor block of right branch (Fig. 3).

Laboratory tests reveal important leukocytosis $\left(22.48 \times 10^{3} / \mathrm{ul}\right)$, thrombocytosis $\left(550 \times 10^{3} / \mathrm{ul}\right)$, mild hypochromic anemia (hemoglobin, $10.2 \mathrm{~g} / \mathrm{dl}$ ) with hyposideremia $(13 \mathrm{ug} / \mathrm{dl})$ and inflammatory syndrome (ESR of $51 \mathrm{~mm} / \mathrm{h}, \mathrm{CRP}$ of $59.50 \mathrm{mg} / \mathrm{L}$ and fibrinogen $538 \mathrm{mg} / \mathrm{dl}$ ); other lab tests are in normal range.

Chest X-ray shows enlarged left pulmonary hilum, minimal vascular and interstitial thickening of the left lower lobe and the pelvic X-ray shows bilateral grade IV sacroileitis and metallic prosthesis of coxofemural joints (Fig. 4).

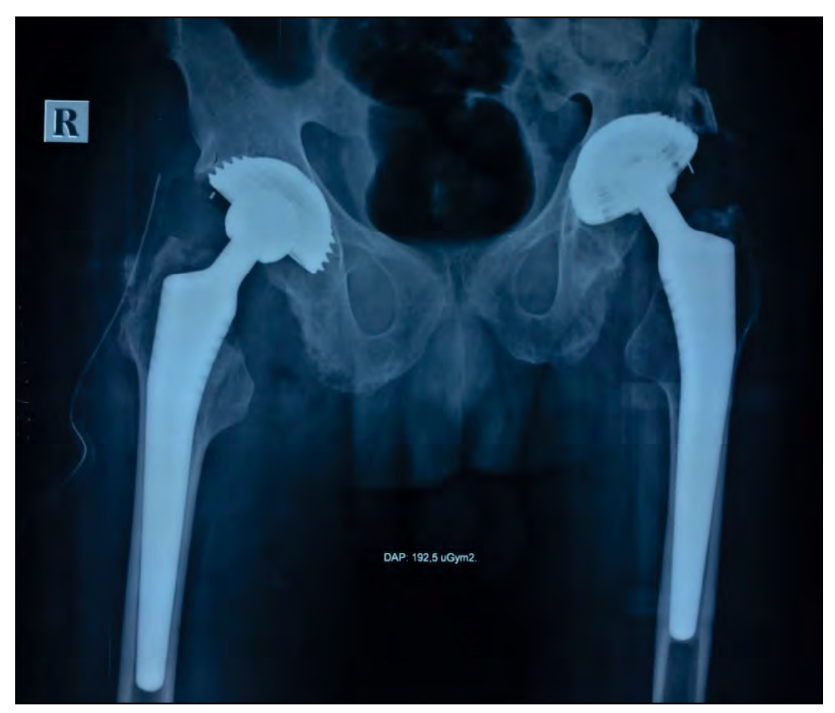

FIGURE 4. Bilateral hip arthroplasty with metallic prostheses

Colonoscopy was performed in 2017 and showed edematous, erythematous mucosa with spontaneous bleeding from the right colic flexure to the cecum and infracentimetric superficial ulcerations. The lesions had a segmental character being interposed with normal mucosal areas. Multiple biopsies were taken at that time, confirming the Crohn's disease (Fig. 5).

The confirmed diagnosis of this HLA B27 patient is axial AS with grade IV bilateral sacroileitis and bilateral metallic prosthesis of coxofemural joints, with early onset and progressive evolution, currently under treatment with golimumab (Simponi). The patient associates histologically confirmed Crohn's disease and Noonan syndrome with typical features and operated congenital cardiac malformation.

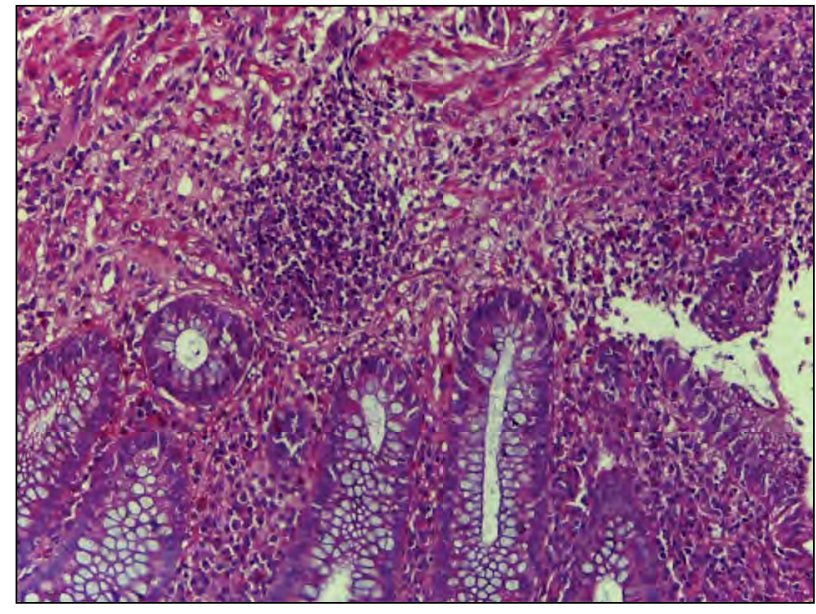

FIGURE 5. Colon mucosa with regenerative changes in the lamina propria and muscularis mucosae, severe acumulation of lymphocytes and plasma cells. Lymphoid aggregates adjacent to blood vessels and lymphatics with granulation tissue. Thickening of muscularis mucosae.

\section{DISCUSSION}

This case is interesting because the association of AS with a genetic disease such as Noonan syndrome is rare and both of these diseases might have complications and comorbidities severely affecting the quality of life. There are few case reports in the medical literature showing the association of Noonan syndrome with peripheral spondyloarthrities and even with acute rheumatic fever $(10,11)$. This association of Noonan syndrome with rheumatic diseases represents a challenge for the clinicians because both of them can affect in different ways the skeleton.

The JOAS of this patient is a severe form of the disease and has a negative prognosis. The evolution of the rheumatologic disease included early coxo-femoral joint damage for which total bilateral hip arthroplasty with metallic prostheses were performed. Considering that the disease did not respond to non-steroidal anti-inflammatory therapy and that the patient had digestive intolerance to sulfasalazine, he was treated with several successive biological therapies (adalimumab, etanercept, infliximab, certolizumab) and was either non-responsive or had allergic reactions (Fig. 6).

During the course of the disease, colonoscopy and biopsy of colon mucosa were performed, histologically confirming Crohn's disease. IBD is generally reported to be associated with spondylarthropathies in 5\%-15\% of cases (12). Studies have shown that microscopic gut inflammation was found in ear- 


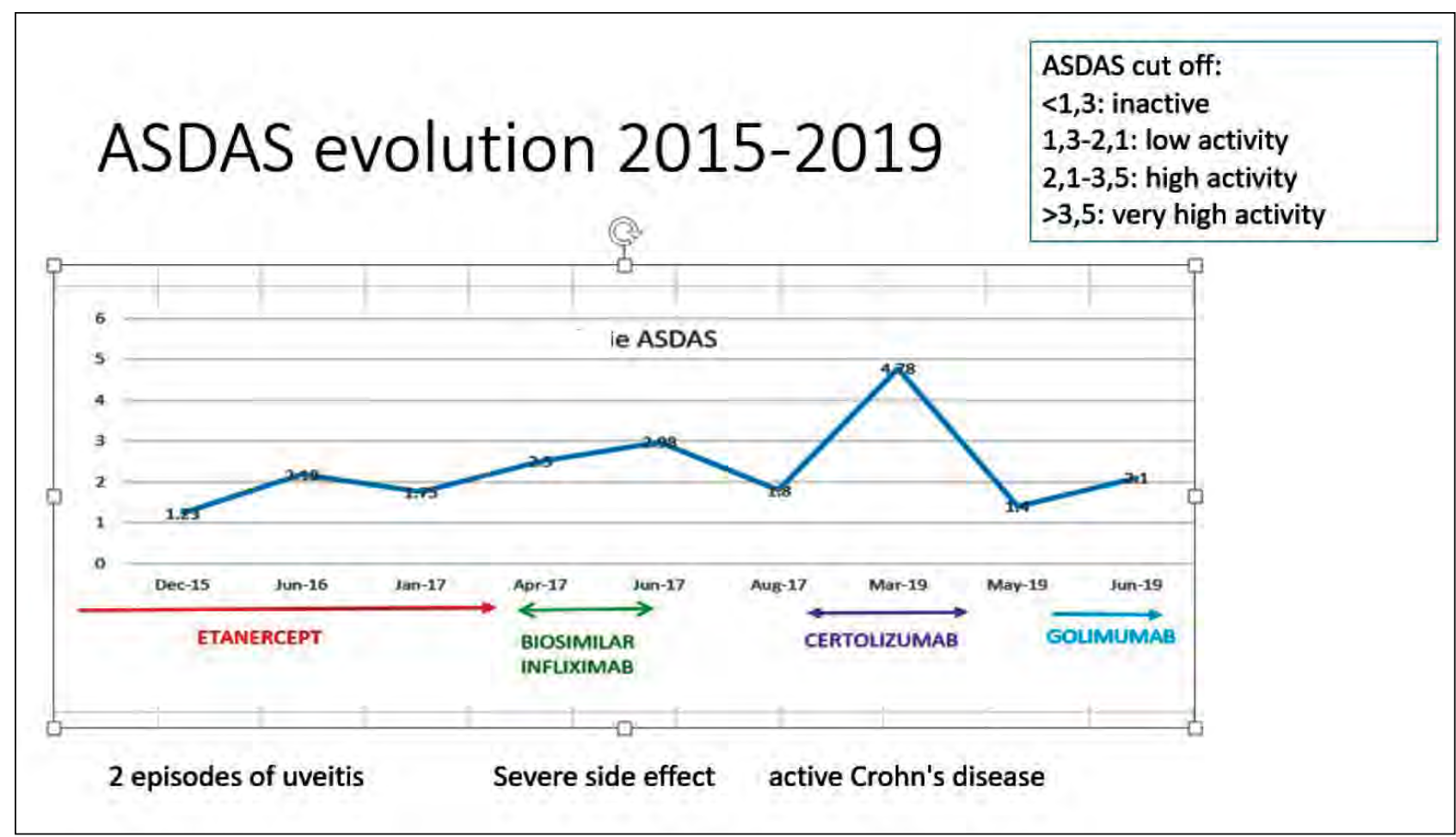

FIGURE 6. Patient's evolution under biological treatment

ly forms of AS and is associated with age, sex, disease activity and degree of MRI inflammation on sacroiliac joints. Althought the connection is further supported by overlapping treatment options for AS and IBD, therapeutic outcomes are not always the same. These dissimilarities can be assigned to differences in not only the cytokine pathways and cells involved in disease, tissue localization and environmental factors but also in pharmacokinetics and biodistribution (13).

Currently, the patient is in treatment with golimumab and budesonide and the short-term progression is favorable.

\section{CONCLUSIONS}

JOAS is a severe disease with predominant peripheral joint involvement, with earlier coxitis de- velopment and a higher predisposition for hip prosthesis. This form of the disease requires multiple non-biological and biological drug treatments to achieve the therapeutic goals. Patients should be closely monitored in the context of comorbidities, disease complications and treatment. The presence of a genetic disease in a patient with JOAS is a challenge in clinical practice. Moreover, the association of JOAS with Crohn's disease is a challenge for both rheumatologists and gastroenterologists. To summarize, the management of this patient with JOAS and Crohn's disease in association with Noonan syndrome implies multidisciplinary health care for a good outcome.

Conflict of interest: none declared Financial support: none declared

\section{REFERENCES}

1. Sieper J, Rudwaleit M, Baraliakos X et al. The Assessment of SpondyloArthritis International Society (ASAS) handbook: A guide to assess spondyloarthritis. Annals of the Rheumatic Diseases. 2009;68(2): ii1-44

2. Proft F, Poddubnyy D. Ankylosing spondylitis and axial spondyloarthritis: Recent insights and impact of new classification criteria. Therapeutic Advances in Musculoskeletal Disease. 2018;10(5-6): 129-139.

3. Deepak RJ, Athimalaipet VR, Raj S. Juvenile Versus Adultonset Ankylosing Spondylitis -Clinical, Radiographic, and Social

Outcomes. A Systematic Review. The Journal of Rheumatology. 2013; 40(11): 1797-1805.

4. Stone M, Warren RW, Bruckel J et al. Juvenile - onset ankylosing spondylitis is associated with worse functional outcomes than adult - onset ankylosing spondylitis. Arthritis \& Rheumatism. 2005;53: 445-451.

5. Mielants $\mathrm{H}$, Veys EM, Cuvelier $\mathrm{C}$ et al. The evolution of spondyloarthropathies in relation to gut histology. II. Histological aspects. The Journal of Rheumatology. 1995;22: 2273-2278. 
6. Miller BS. The History of Noonan Syndrome. Pediatric Endocrinology Reviews 2019;16:424-427.

7. Rohrer T. Noonan Syndrome: Introduction and Basic Clinical Features. Horm Res. 2009;72(2): 3-7.

8. Allanson JE, Roberts AE. Noonan Syndrome. GeneReviews ${ }^{\circledR}$ (Seattle WA). 1993.

9. Chen PC, Yin J, Yu HW et al. Next-generation sequencing identifies rare variants associated with Noonan syndrome. Proc Natl Acad Sci U S A. 2014;111(31): 11473-11478.

10. Saldarriaga Rivera LM, Fernandes de Melo E, Damião Araujo $\mathrm{P}$ et al. Peripheral spondyloarthritis in a patient with Noonan's syndrome. Reumatol Clin 2015; 11(2): 112-115.
11. Tramboo NA, lqbal $K$, Malik AR et al. Acute rheumatic fever in Noonan syndrome. Indian J Pediatr. 2000; 67(8): 605-607.

12. Kopylov U, Starr M, Watts $C$ et al. Detection of Crohn Disease in Patients with Spondyloarthropathy: The SpACE Capsule Study. The Journal of Rheumatology. 2018; 45(4): 498-505.

13. De Wilde K, Debusschere K, Beeckman S et al. Integrating the pathogenesis of spondyloarthritis: gut and joint united? Current Opinion in Rheumatology 2015;27(2): 189-196. 